\title{
Massive pneumoperitoneum during endoscopic ultrasound-guided drainage of a pancreatic cyst lesion, treated with an enteral self-expanding metal stent and paracentesis
}

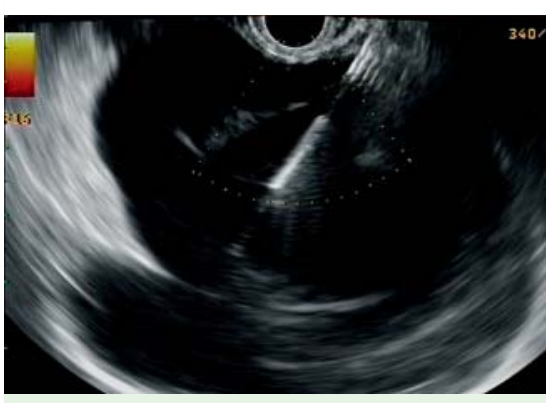

Fig. 1 Endoscopic ultrasound (EUS) image of a cystic lesion located in the pancreatic tail.

A 54-year-old patient with a supposed 9-cm pseudocyst located in the pancreatic tail was referred to our unit for endoscopic ultrasound (EUS)-guided transmural drainage ( Fig.1). The fluoroscopic image showed the immediate creation of a pneumoperitoneum when the cystotome was used, because the cystic lesion was not adherent to the gastric wall ( $\bullet$ Fig.2a). First, we attempted to seal the ostomy (or iatrogenic perforation), with the purpose of preventing leakage of fluids to the peritoneal cavity, by using a 'diabolo'-shaped self-expanding metal stent (SEMS) (AXIOS $10 \mathrm{~mm} \times 10 \mathrm{~mm}$; Xlumena, Mountain View, California, USA), without success ( $\bullet$ Fig. 2 b). Finally, a successful rescue maneuver was done in which a fully covered SEMS (60 mm× $16 \mathrm{~mm}$, Hanaro; MI-Tech, Seoul, Korea) was delivered coaxially with the first and migrated stent ( $\bullet$ Fig.3a,b). Computed tomography (CT) showed a sealed perforation without leakage of fluid, and a massive pneumoperitoneum ( Fig.4, - Video 1).

The patient had a favorable outcome from decompression paracentesis. The cystic fluid analysis showed high carcinoembryonic antigen (CEA) levels, identifying

\section{Video 1}

Massive pneumoperitoneum during endoscopic ultrasound (EUS)-guided drainage of a pancreatic cyst lesion, treated by using an enteral fully covered self-expanding metal stent (FC-SEMS).

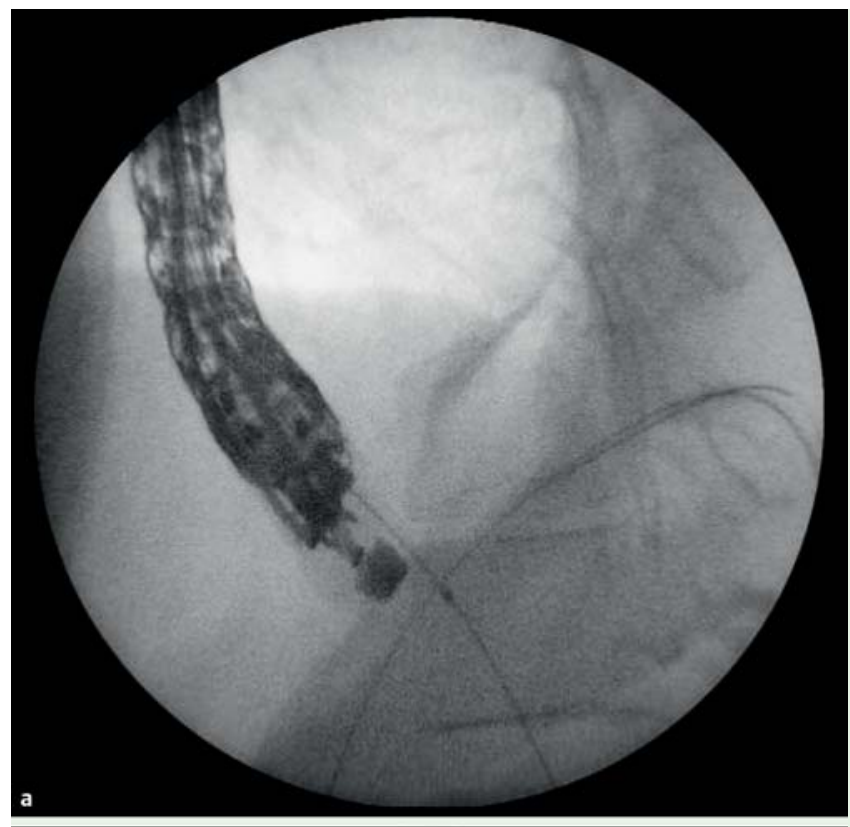

Fig. 2 Fluoroscopic images showing: a Creation of a pneumoperitoneum caused by the use of a cystotome in a pancreatic lesion that was not adherent to the gastric wall. b Failed attempt to seal the perforation using a 'diabolo'shaped self-expanding metal stent (SEMS).

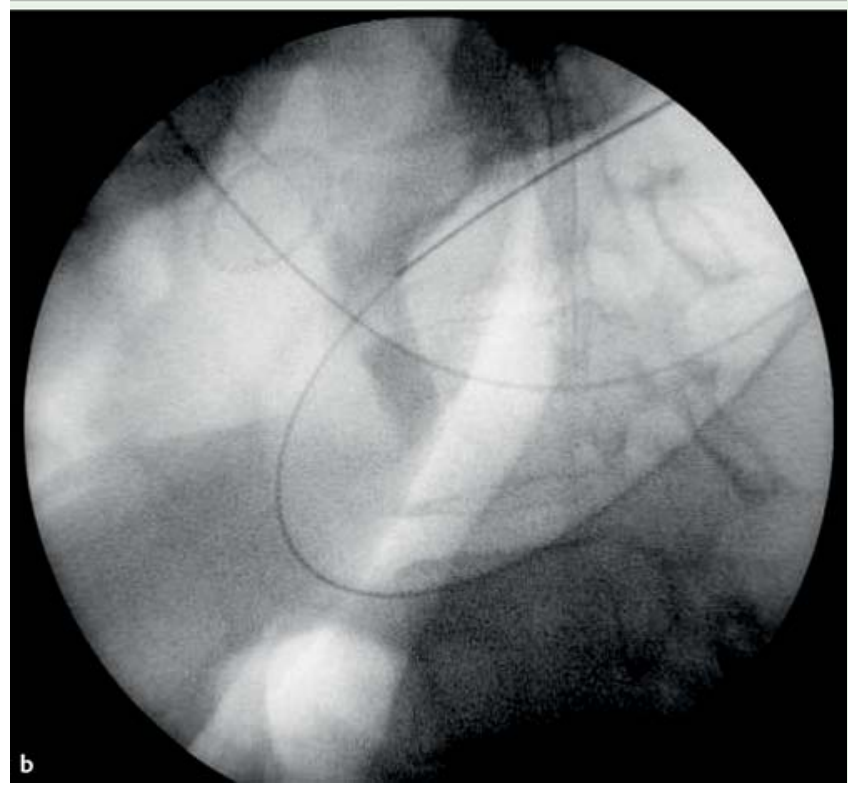

Even so, before drainage, evaluation of the lesion is necessary to rule out intervening vessels, to determine the adherence of the lesion, and to confirm that the nature of the lesion is other than inflammatory (i.e., a cystic tumor).

The present report emphasizes the point that excellent pre-assessment is essential to avoid the mistake of confusing a cystic neoplasm with a pseudocyst [1-3]. 

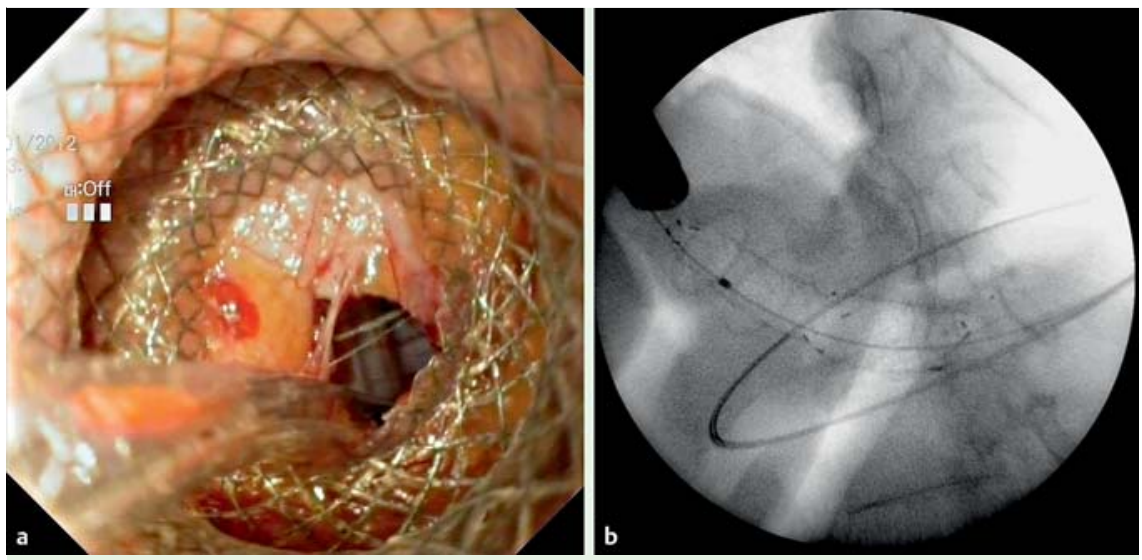

Fig. 3 a Endoscopic view through the stent of the perforation, and a catheter balloon. b Fluoroscopic image of the stent-in-stent rescue maneuver: a longer enteral fully covered self-expanding metal stent (FC-SEMS) was delivered coaxially with the first (and migrated) stent.

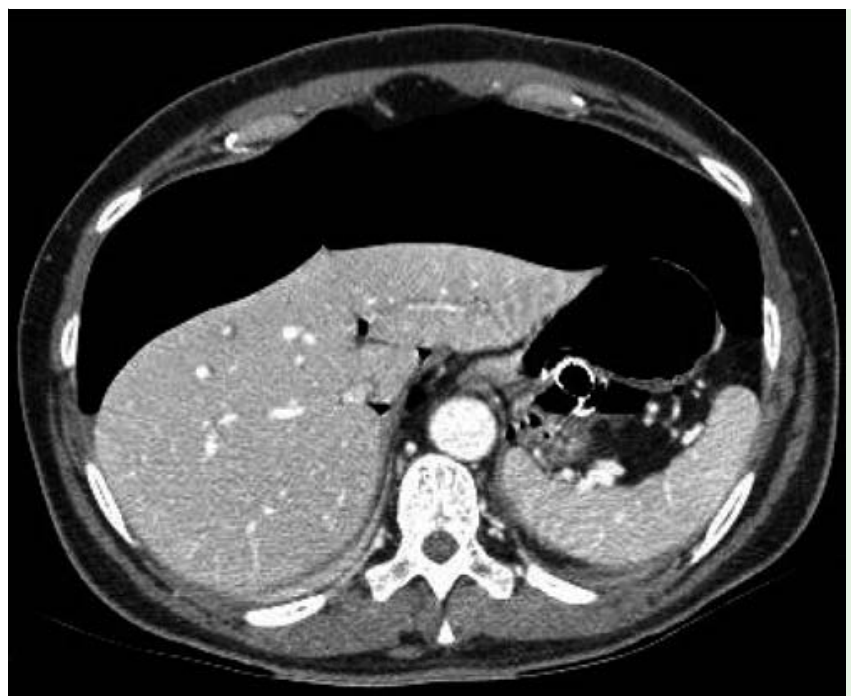

Fig.4 Emergency computed tomography (CT) showed that the perforation was well sealed without leakage of fluid, and a massive pneumoperitoneum.

In the case of massive pneumoperitoneum, the previously described rescue technique, of delivering a long enteral FC-SEMS and performing an emergency paracentesis, can be helpful $[4,5]$.

Endoscopy_UCTN_Code_CPL_1AL_2AD

Competing interests: None
Claudia Consiglieri ${ }^{1}$, Joan B. Gornals ${ }^{1}$, Carlos Huertas ${ }^{1}$, Zoilo Madrazo², Carme Loras ${ }^{1}$

${ }^{1}$ Endoscopy Unit, Department of Digestive Diseases, Hospital Universitari de Bellvitge-IDIBELL, Barcelona, Spain

2 Department of Surgery, Hospital Universitari de Bellvitge-IDIBELL, Barcelona, Spain

\section{References}

1 Ahmad NA, Kochman ML, Brensinger C et al Interobserver agreement among endosonographers for the diagnosis of neoplastic versus non-neoplastic pancreatic cystic lesions. Gastrointest Endosc 2003; 58: 59-64

2 Gintowt A, Hac S, Dobrowolski $S$ et al. An unusual presentation of pancreatic pseudocyst mimicking cystic neoplasm of the pancreas: a case report. Cases J 2009; 2: 9138

3 Weilert F, Binmoeller KF, Shah JN et al. Endoscopic ultrasound-guided drainage of pancreatic fluid collections with indeterminate adherence using temporary covered metal stents. Endoscopy 2012; 44: 780 - 783

4 Chiapponi C, Stocker U, Körner M et al. Emergency percutaneous needle decompression for tension pneumoperitoneum. BMC Gastroenterol 2011; $11: 48$

5 Andrews AH, Horwhat JD. Massive pneumoperitoneum after EUS-FNA aspiration of the pancreas. Gastrointest Endosc 2006; 63: 876-877

Bibliography

DOI http://dx.doi.org/

10.1055/s-0034-1377222

Endoscopy 2014; 46: E330-E331

(C) Georg Thieme Verlag KG

Stuttgart · New York

ISSN 0013-726X

\section{Corresponding author}

\section{Joan B. Gornals, MD, PhD}

Endoscopy Unit, Department of Digestive Diseases Hospital Universitari de Bellvitge-IDIBELL

(Bellvitge Biomedical Research Institute)

Feixa Llarga s/n

08907 L'Hospitalet de Llobregat

Barcelona

Spain

Fax: +34-93-2607681

jgornals@bellvitgehospital.cat 